\title{
Compartilhar o leito com cão: impacto no sono do dono
}

\author{
Sharing bed with dog: impact in owner's sleep
}

\author{
Bruna do Prado Bertocco ${ }^{1}$, Lucila Bizari Fernandes do Prado², Gilmar \\ Fernandes do Prado ${ }^{3}$, Luciane Bizari Coin de Carvalho ${ }^{4}$
}

1.Médica Veterinária, Mestre, Setor Neuro-Sono, Disciplina de Neurologia Clínica, Escola Paulista de Medicina, Universidade Federal de São Paulo, São Paulo, SP, Brasil.

2.Médica, Doutora, Setor Neuro-Sono, Disciplina de Neurologia Clínica, Escola Paulista de Medicina, Universidade Federal de São Paulo, São Paulo, SP, Brasil.

3.Neurologista, Doutor, Professor Adjunto, Setor Neuro-Sono, Disciplina de Neurologia Clínica, Escola Paulista de Medicina, Universidade Federal de São Paulo, São Paulo, SP, Brasil.

4.Pscóloga, Doutora, Professora Afiliada, Setor Neuro-Sono, Disciplina de Neurologia Clínica, Escola Paulista de Medicina, Universidade Federal de São Paulo, São Paulo, SP, Brasil.

\begin{abstract}
Resumo
Objetivo. Verificar se dormir junto com um cachorro influencia o sono do seu dono. Método. Foram entrevistados 549 proprietários de cães e 273 indivíduos que não possuíam animais de estimação (grupo controle). Os proprietários de cães entrevistados foram divididos em: dormir com cão no leito, dormir com o cão no quarto (em outro lugar que não o leito), dormir com cão na casa (cão dorme em outro lugar da casa). Resultados. Dos 549 participantes da pesquisa, 56\% (312) eram da faixa etária entre 19 a 30 anos, 53\% (293) eram do sexo feminino, 50\% (270) eram solteiros e 53\% (290) possuíam nível superior completo. A quantidade de proprietários do grupo leito e quarto, que relatou que seu sono não foi prejudicado por dormir com o cão foi maior quando comparada a quantidade de proprietários do grupo sem cão $(47 \% ; 49 \% ; 35 \%$ respectivamente; $\mathrm{p}<0,05)$. As mulheres, com escolaridade superior e sem queixas de sono que dormiam com o cão apresentaram mais chance de considerar seu sono não prejudicado por dormir com o cão. O grupo sem cão apresentou maior quantidade de sujeitos com nenhum despertar $(18 \%)$ em relação aos grupos leito $(8 \%)$, quarto (zero \%) e casa $(5 \%, p<0,05)$. Conclusão. Os proprietários que dormem com o cão no leito ou no quarto consideram seu sono não prejudicado e queixam-se menos de problemas de sono, apesar de apresentarem despertares.
\end{abstract}

Unitermos. Compartilhamento de leito; sono de cães; impacto no sono

\begin{abstract}
Objective. Assessing whether sleeping with a dog influences its owner's sleep. Method. 549 dog owners and 273 non-pet owners (control group) were interviewed. Dog owners interviewed were divided into: sleeping with dog in bed, sleeping with dog in bedroom (other than bed), sleeping with dog in house (dog sleeps elsewhere in house). Results. Of the 549 participants, 56\% (312) were 19 to 30 years old, 53\% (293) were female, 50\% (270) were single, and $53 \%(290)$ had college. The number of owners of bed and bedroom group who reported that their sleep was not impaired by sleeping with the dog was higher when compared to the number of owners of no dog group (47\%; $49 \% ; 35 \%$ respectively; $\mathrm{p}<0.05)$. Women with higher education and no complaints of sleep sleeping with the dog were more likely to consider their sleep not harmed by sleeping with the dog. The group without dogs had more subjects with no awakening (18\%) compared to bed $(8 \%)$, bedroom (zero\%) and home $(5 \%, \mathrm{p}<0.05)$ groups. Conclusion. Owners who sleep with the dog in bed or bedroom find their sleep had not changed and complain less about sleep problems, although they do have awakenings.
\end{abstract}

Keywords. Stroke; Sleep; Sleep Disorders

Trabalho realizado no Setor Neuro-Sono, Disciplina de Neurologia Clínica, Escola Paulista de Medicina, Universidade Federal de São Paulo, São Paulo, SP, Brasil. 


\section{INTRODUÇÃO}

Domesticados pelo homem desde a antiguidade para auxiliar na caça, guarda, proteção e pastoreio, hoje os cães ganharam o status de "membros da família". Nesse vínculo com o ser humano é comum que compartilhem o leito com seus proprietários ${ }^{1,2}$.

O compatilhamento de leito vem sendo objeto de interesse científico, pois tanto os benefícios quanto os possíveis problemas dessa prática ainda não estão bem definidos $^{3}$. O compartilhamento do leito de pais com bebês pode ser uma prática importante em algumas culturas, mas pode aumentar a chance de morte súbita na infância ${ }^{4}$. Para casais que compartilham o leito durante o sono, a movimentação de um pode incomodar o outro parceiro, levando a uma qualidade de sono ruim 5 .

A Comissão de Animais de Companhia (COMAC) do Sindicato Nacional da Indústria de Produtos para Saúde Animal (SINDAN), Brasil, mostrou que $55 \%$ dos cães de estimação dormem dentro de casa e $23 \%$ dentro do quarto dos donos ${ }^{6}$. Em estudo preliminar, encontramos $42 \%$ de proprietários compartilhavam o quarto com seus cães e $62 \%$ destes também compartilhavam o leito ${ }^{7}$. Compartilhar o leito com cães pode beneficiar o sono do proprietário, da 
mesma forma que o convívio com eles promovem benefícios psicofisiológicos, porém esta prática pode trazer prejuízos como a transmissão de doenças, desenvolvimento de alergias e dermatites ${ }^{8}$, mas não se sabe o real prejuízo ao sono do proprietário e do cão.

Em vista da ausência de dados sobre o impacto que o compartilhamento do leito com cães exerce sobre o sono do proprietário, o objetivo desse estudo foi verificar se a qualidade subjetiva do sono, o número de despertares noturno e as queixas sobre problemas relacionados ao sono são afetados por esse comportamento.

\section{MÉTODO}

\section{Amostra}

Foram entrevistadas 549 pessoas consecutivamente no período de julho a outubro de 2012. Foram 276 proprietários de cães e 273 indivíduos que não possuíam animais de estimação, pareados pela idade do proprietário.

A amostra foi calculada considerando-se a prevalência de $78 \%$ de proprietários de cães na cidade de São Paulo ${ }^{6}$, resultando em um valor de 546 proprietários. Foram excluídos da pesquisa proprietários que compartilhavam o leito com cães e gatos ao mesmo tempo ou proprietários que relataram compartilhar o leito com outras espécies animais. A faixa etária dos entrevistados variou de 14 a 70 anos.

Este estudo foi aprovado pelo Comitê de Ética em 
Pesquisa da Universidade Federal de São Paulo, Escola Paulista de Medicia (CAAE: 02421812.7.0000.5505).

\section{Procedimento}

Foram realizadas entrevistas semi-estruturadas, nos parques públicos da cidade de São Paulo, por um dos pesquisadores (BPB), que convidava os frequentadores do local a participarem da pesquisa. Cada entrevista não excedeu 5 minutos.

Grupo Estudo. Os proprietários de cães de estimação responderam questões sobre o número de cães presentes na residência, temperamento comportamental do cão, o local onde o cão dormia (na mesma cama que seu proprietário, no mesmo quarto, ou outro local da residência), rotina de sono e de horários do proprietário e do cachorro, se o cão pedia para sair do quarto durante o período de sono, o número de despertares durante 0 período de sono que este proprietário atribuía ao cão.

O proprietário deveria avaliar o impacto da presença do cão na casa, no quarto ou no leito sobre seu sono, se atrapalhava ou não. Quando o proprietário respondia positivamente a essa pergunta, consideramos que o sono era prejudicado pelo animal. Obteve-se também uma breve história clínica do cão, buscando-se saber sobre a rotina da vacinação e vermifugação, frequência com que ia ao veterinário e se possuía ectoparasitas como pulgas, carrapatos e ácaros.

Grupo Controle. Os entrevistados que não possuíam 
nenhum animal de estimação responderam sobre horários e rotinas do sono, número de despertares e por quais motivos, se alguma vez já possuíram um cão de estimação e se compartilhavam o leito com ele, e se possuía alguma queixa sobre seu sono.

\section{Análise das Variáveis}

Os proprietários de cães foram classificados segundo o local em que o cão dormia, em: 1) casa (cão dorme em outro lugar da casa que não o quarto), 2) quarto (cão dorme em algum lugar do quarto menos o leito), 3) leito (cão dorme com proprietário no leito). Esses grupos foram comparados entre si e ao grupo sem cão.

As variáveis avaliadas foram impacto do cão no sono do proprietário (prejudica, não prejudica), número de despertares do proprietário durante o período de sono (nenhum, um ou mais), presença de alguma queixa de sono (sim, não), horário em que o proprietário dormia (antes da meia noite e depois da meia noite), horário que o proprietário acordava (antes das sete horas e depois das sete horas). As variáveis foram analisadas segundo a faixa etária dos entrevistados ( 0 a 12 anos, 13 a 18 anos, 19 a 30 anos, 31 a 40 anos, 41 a 50 anos, 51 a 60 anos, 61 a 70 anos, mais de 70 anos), sexo (feminino, masculino), estado civil (solteiro, casado) e escolaridade (ensino fundamental incompleto, ensino fundamental completo, ensino médio incompleto, ensino médio completo, ensino superior incompleto, ensino superior completo). 


\section{Análise Estatística}

Utilizamos os testes de Qui-Quadrado e Exato de Fisher para analisar as variáveis categorias, o teste $\mathrm{t}$ de Student para as variáveis numéricas e o teste de Regressão Logística para verificar a interação das variáveis, adotandose o nível de significância de $p<0,05$.

\section{RESULTADOS}

\section{Dados Demográficos}

Dos 549 participantes da pesquisa, 56\% (312) estavam da faixa etária entre 19 a 30 anos, 53\% (293) eram do sexo feminino, $50 \%$ (270) eram solteiros e $53 \%$ (290) possuíam nível superior completo. Em relação aos cães, 63\% (178) eram fêmeas, com idade entre 2 a 5 anos, $42 \%$ (118) eram de pequeno porte, e 51\% (142) não eram castrados. Todos os proprietários consideraram seu cão com temperamento dócil e brincalhão, frequentavam regularmente o médico veterinário, possuíam vermifugação e vacinação em dia.

Dos 276 participantes que eram proprietários de cães, $36 \%$ eram do grupo leito, $25 \%$ do grupo quarto e $39 \%$ do grupo casa (Tabela 1 ).

Tabela 1. Dados Demográficos dos participantes dos grupos dormir com cão, dormir sem cão e grupo controle (não tem cão). 


\begin{tabular}{|c|c|c|c|c|}
\hline & \multicolumn{3}{|c|}{$\begin{array}{c}\text { Tem cão } \\
276(100)\end{array}$} & \multirow[b]{2}{*}{$\begin{array}{l}\text { Sem cão } \\
273(100)\end{array}$} \\
\hline & $\begin{array}{c}\text { Dorme no } \\
\text { leito } \\
99(36)\end{array}$ & $\begin{array}{c}\text { Dorme } \\
\text { no } \\
\text { quarto } \\
70(25) \\
\end{array}$ & $\begin{array}{l}\text { Dorme } \\
\text { em casa } \\
107(39)\end{array}$ & \\
\hline \multicolumn{5}{|l|}{ Idade } \\
\hline $\begin{array}{c}0 \text { a } 12 \text { anos } \\
13 \text { a } 18 \text { anos } \\
19 \text { a } 30 \text { anos } \\
31 \text { a } 40 \text { anos } \\
41 \text { a } 50 \text { anos } \\
51 \text { a } 60 \text { anos } \\
61 \text { a } 70 \text { anos } \\
\text { mais de } 70 \text { anos }\end{array}$ & $\begin{array}{c}0 \\
19(19)^{\mathrm{a}} \\
41(41)^{\mathrm{b}, \mathrm{d}} \\
22(22)^{\mathrm{a}, \mathrm{b}, \mathrm{d}} \\
0 \\
5(5) \\
8(8) \\
4(4)\end{array}$ & $\begin{array}{c}0 \\
1(1)^{\mathrm{c}, \mathrm{e}} \\
34(49)^{\mathrm{e}} \\
26(37)^{\mathrm{c}, \mathrm{e}} \\
1(1) \\
2(3) \\
2(3) \\
4(6)\end{array}$ & $\begin{array}{c}0 \\
13(12) \\
66(62) \\
6(6) \\
1(1) \\
6(6) \\
9(8) \\
6(6)\end{array}$ & $\begin{array}{c}0 \\
34(12) \\
171(63) \\
18(7) \\
2(1) \\
15(5) \\
19(7) \\
14(5)\end{array}$ \\
\hline \multicolumn{5}{|l|}{ Sexo } \\
\hline $\begin{array}{c}\begin{array}{c}\text { Feminino } \\
\text { Masculino }\end{array} \\
\end{array}$ & $\begin{array}{l}58(59) \\
41(41) \\
\end{array}$ & $\begin{array}{l}32(46) \\
38(54)\end{array}$ & $\begin{array}{l}58(54) \\
49(46)\end{array}$ & $\begin{array}{l}145(53) \\
128(47)\end{array}$ \\
\hline \multicolumn{5}{|l|}{ Estado Civil } \\
\hline $\begin{array}{c}\text { Solteiro } \\
\text { Casado }\end{array}$ & $\begin{array}{l}57(58)^{a} \\
42(42)\end{array}$ & $\begin{array}{l}29(41) \\
41(59)\end{array}$ & $\begin{array}{l}50(47) \\
57(53)\end{array}$ & $\begin{array}{l}142(52) \\
131(48)\end{array}$ \\
\hline \multicolumn{5}{|l|}{ Escolaridade } \\
\hline $\begin{array}{l}\text { Ensino fundamental } \\
\text { incompleto }\end{array}$ & $2(2)^{d}$ & $2(3)^{e}$ & 0 & 0 \\
\hline $\begin{array}{c}\text { Ensino fundamental } \\
\text { completo }\end{array}$ & $1(1)^{a}$ & $8(11)^{c, e}$ & 0 & 0 \\
\hline $\begin{array}{l}\text { Ensino médio } \\
\text { incompleto }\end{array}$ & $10(10)^{\mathrm{a}, \mathrm{c}}$ & $1(1)^{e}$ & $12(11)$ & $33(12)$ \\
\hline $\begin{array}{l}\text { Ensino médio } \\
\text { completo }\end{array}$ & $16(16)^{a, d}$ & $3(4)$ & $9(8)$ & $24(9)$ \\
\hline $\begin{array}{l}\text { Ensino superior } \\
\text { incompleto }\end{array}$ & $27(27)^{a}$ & $9(13)^{\mathrm{c}, \mathrm{e}}$ & $29(27)$ & $73(27)$ \\
\hline $\begin{array}{l}\text { Ensino superior } \\
\text { completo }\end{array}$ & $43(43)^{a}$ & $47(67)^{e}$ & $57(53)$ & $143(52)$ \\
\hline
\end{tabular}

A maioria dos proprietários do grupo leito e quarto estava nas faixas etárias de 13 a 18 anos, 19 a 30 anos e 31 a 40 anos ( $p<0,05 ;$ Tabela 1$)$. Houve tendência a ter mais mulheres do grupo leito do que do grupo quarto (59\%, 46\% respectivamente; $p<0,05)$. Foi observada uma maior quantidade de proprietários solteiros do grupo leito em comparação ao grupo quarto (58\%, $41 \%$ 
respectivamente; $p<0,05)$. Havia mais proprietários com ensino médio no grupo leito do que os outros grupos $(p<0,05)$. Já os de ensino superior, eram do grupo leito ou quarto do que os grupos casa e sem cão $(p<0,05)$.

\section{Impacto do cão no sono do proprietário}

A quantidade de proprietários do grupo leito e que relatou que o cão não tinha impacto sobre seu sono foi semelhante quando comparada aos grupos dormir quarto e casa, mas foi maior quando comparada a quantidade de pessoas do grupo sem cão (47\%; 35\% respectivamente; $p<0,05$; Tabela 2). O grupo quarto também apresentou maior quantidade de proprietários que consideraram que 0 cão não prejudicava seu sono em relação ao grupo sem cão (49\%, 35\%; respectivamente; $p<0,05)$.

As variáveis sexo, escolaridade e problemas de sono foram significantes na interação que explica o impacto do cão no sono do proprietário e foram incluídas no modelo de Regressão Logística. As mulheres com escolaridade fundamental ou ensino médio e sem queixa sobre problemas de sono apresentaram chance de 1,021 vezes a mais de considerar que seu cão não prejudica seu sono em relação aos homens com as mesmas características. As mulheres com escolaridade superior e sem queixa sobre problemas de sono apresentaram chance de 1,034 a mais de considerar que seu cão não prejudica seu sono,

Tabela 2. Distribuição dos participantes nas variáveis observadas. 
$276(100)$

\begin{tabular}{|c|c|c|c|c|}
\hline & $\begin{array}{l}\text { Dorme no } \\
\text { leito } \\
99(36) \\
\end{array}$ & $\begin{array}{c}\text { Dorme no } \\
\text { quarto } \\
70(25) \\
\end{array}$ & $\begin{array}{c}\text { Dorme na } \\
\text { casa } \\
107(39)\end{array}$ & $\begin{array}{l}\text { Sem cão } \\
273(100)\end{array}$ \\
\hline $\begin{array}{c}\text { Impacto sono } \\
\text { Não tem prejuízo } \\
\text { Tem prejuízo }\end{array}$ & $\begin{array}{l}47(47)^{d} \\
52(53)\end{array}$ & $\begin{array}{l}34(49)^{\mathrm{e}} \\
36(51)\end{array}$ & $\begin{array}{l}37(35) \\
70(65) \\
\end{array}$ & $\begin{array}{c}96(35) \\
177(65) \\
\end{array}$ \\
\hline $\begin{array}{l}\text { Despertares } \\
\text { Nenhum } \\
\text { Um ou mais }\end{array}$ & $\begin{array}{l}8(8)^{a, d} \\
91(92)\end{array}$ & $\begin{array}{c}0^{\mathrm{e}} \\
70(100)\end{array}$ & $\begin{array}{c}5(5)^{f} \\
102(95)\end{array}$ & $\begin{array}{c}49(18) \\
224(82)\end{array}$ \\
\hline $\begin{array}{l}\text { Horário que dorme } \\
\text { Antes das 00:00 hs } \\
\text { Após as 00:00 hs }\end{array}$ & $\begin{array}{c}84(85)^{a, b, d} \\
15(15)\end{array}$ & $\begin{array}{l}42(60)^{\mathrm{e}} \\
28(40)\end{array}$ & $\begin{array}{l}74(69) \\
33(31)\end{array}$ & $\begin{array}{c}197(72) \\
76(28)\end{array}$ \\
\hline $\begin{array}{l}\text { Horário que acorda } \\
\text { Antes das 7:00 hs } \\
\text { Após as 7:00 hs }\end{array}$ & $\begin{array}{c}95(96)^{b, d} \\
4(4)\end{array}$ & $\begin{array}{c}70(100)^{\mathrm{c}, \mathrm{e}} \\
0 \\
\end{array}$ & $\begin{array}{l}71(66) \\
36(34) \\
\end{array}$ & $\begin{array}{c}184(67) \\
89(33)\end{array}$ \\
\hline $\begin{array}{c}\text { Queixa de } \\
\text { problemas de Sono } \\
\text { Sim } \\
\text { Não }\end{array}$ & $\begin{array}{c}15(15)^{a, b} \\
84(85)\end{array}$ & $\begin{array}{l}48(69)^{c, e} \\
22(31)\end{array}$ & $\begin{array}{l}46(43)^{f} \\
61(57)\end{array}$ & $\begin{array}{c}24(9) \\
249(91)\end{array}$ \\
\hline
\end{tabular}

comparadas aos homens com as mesmas características. As mulheres com escolaridade superior e que se queixaram de problemas de sono, apresentaram chance de 2,064 a mais de considerar que seu cão não prejudica seu sono, comparadas com mulheres com mesmas características e que dormem sem o cão (Tabela 3).

\section{Despertares}

O grupo sem cão apresentou maior quantidade de sujeitos sem nenhum despertar (18\%) em relação aos grupos leito ( $8 \%)$, quarto (zero\%) e casa ( $5 \%$; $p<0,05$; Tabela 2). Não houve diferença no número de despertares em relação ao sexo, idade, estado civil e escolaridade. 
Tabela 3. Chances dos indivíduos participantes da entrevista de não terem o sono prejudicado dormindo com seu cão.

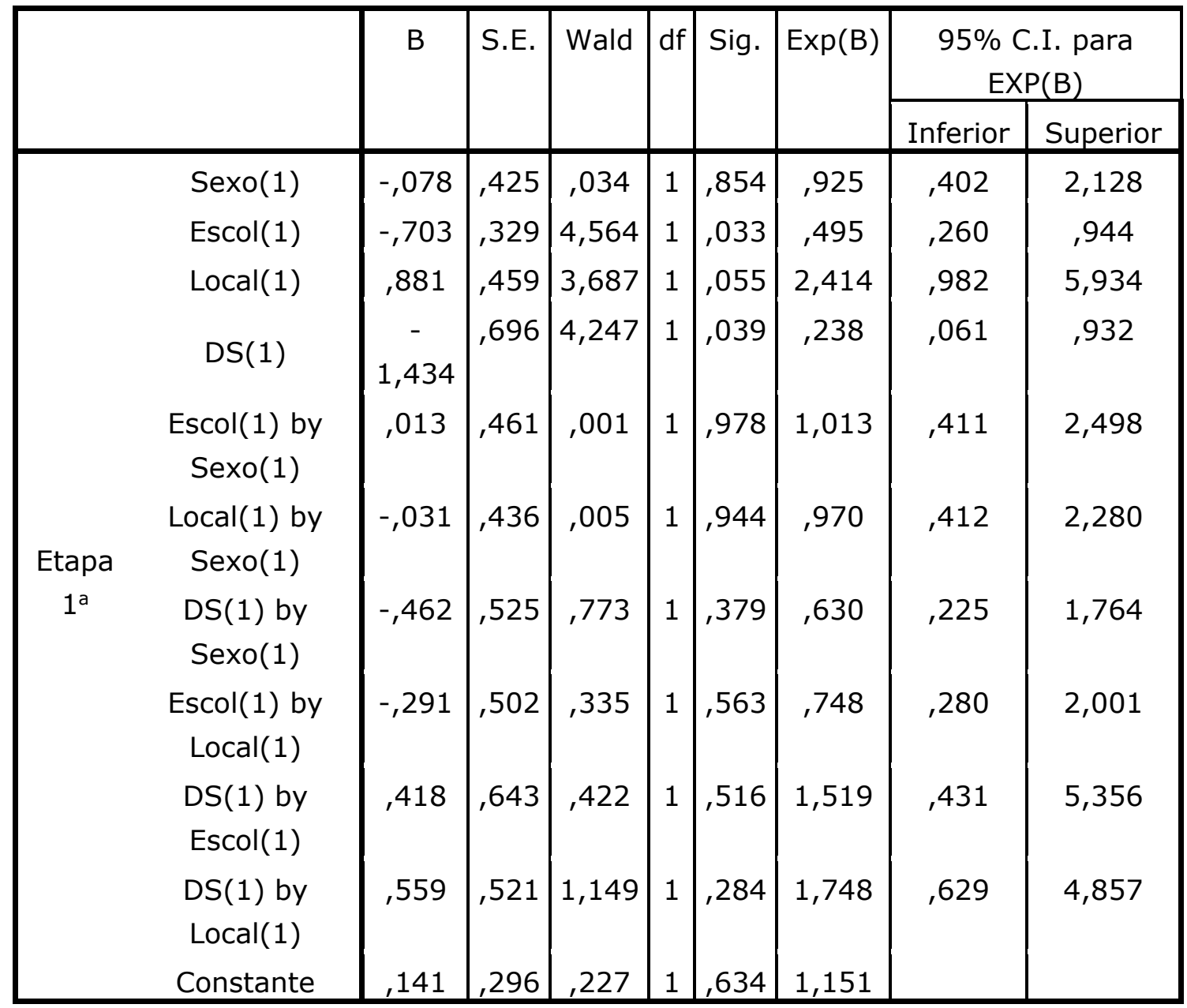

a. Variáveis inseridas na etapa 1: Sexo, Escol, Local, DS, Escol * Sexo, Local * Sexo, DS * Sexo , Escol * Local , DS * Escol , DS * Local.

\section{Problemas de Sono}

O grupo leito apresentou menor quantidade de sujeitos com queixa de distúrbios do sono (15\%) em relação aos grupos quarto $(69 \%)$, casa $(43 \%)$ e sem cão (33\%; $\mathrm{p}<0,05 ;$ Tabela 2). Não houve diferença na queixa de problemas de sono em relação ao sexo, idade, estado civil e escolaridade.

\section{Horário de Dormir e Acordar}


Uma maior quantidade de proprietários do grupo leito (85\%) dormia antes das $24 \mathrm{~h}$, em comparação ao grupo quarto $(60 \%)$, casa $(69 \%)$ e ao grupo sem cão $(72 \%)$ $(p<0,05 ;$ Tabela 2$)$.

Uma maior quantidade de proprietários do grupo leito (96\%) e quarto (100\%) acordavam antes das $7 \mathrm{~h}$, em relação aos grupos casa (66\%) e sem cão $(67 \% ; p<0,05$; Tabela 2).

Não houve diferença entre os horários de dormir e acordar em relação ao sexo, idade, escolaridade e estado civil (Tabela 2).

\section{DISCUSSÃO}

Este é o primeiro estudo que avalia o sono de proprietários que compartilham o leito com seus cães. Os proprietários relataram sono não prejudicado por dormirem com seus cães no leito ou no quarto, apesar de relatarem despertares pela presença do animal e queixas de problemas relacionados ao sono. Os proprietários que apresentaram queixas de sono, não associaram o fato de dormirem com seus cães no leito ou no quarto a um sono ruim ou à presença de despertares.

Compartilhar o leito com um cão implica em aumento de despertares do proprietário e diminuição da eficiência do sono ${ }^{9-12}$, por causa dos ciclos de sono mais curtos dos cães quando comparados ao do humano ${ }^{13}$, mas isso não foi suficiente para o proprietário considerar seu sono ruim. A 
presença do cão dormindo no leito ou no quarto contribuiu positivamente para com o sono do proprietário, semelhante ao observado entre casais que compartilham o leito, mesmo havendo maior número de despertares ${ }^{11,14,15}$. As mulheres que relataram alguma queixa sobre seu sono apresentaram chance maior de considerarem seu sono bom dormindo com seu cão quando comparadas aos homens. Dormir com o cão pode representar um vínculo seguro, favorecendo a percepção de um sono reparador ${ }^{11,14,15}$.

O relacionamento entre os seres humanos e os cães vem se transformando e tem sido cada vez mais afetivo ${ }^{16-}$ 18. A convivência de seres humanos e cães no âmbito da saúde, também têm apresentado uma crescente utilização desse relacionamento como forma terapêutica ${ }^{8,17,19-22}$, o que nos faz pensar se esse convívio contribui para uma mudança cultural no status desse relacionamento ${ }^{19,20}$.

Compartilhar o leito com cães é controverso, havendo especialistas que não recomendam este comportamento, não havendo, entretanto, dados convincentes para tal orientação. Mesmo havendo diversas advertências quanto a esta prática, as pessoas cada vez mais compartilham o leito com seus cães ${ }^{20-23}$.

Não há estudos sugerindo que dormir com cães promova distúrbios do sono no proprietário. São necessárias pesquisas desenhadas especificamente para avaliar os riscos e benefícios ao sono dos proprietários que compartilham suas camas e ambiente de sono com seus cães. Nesse estudo, a avaliação do impacto do cão no sono 
do proprietário foi avaliada por respostas subjetivas, daqueles proprietários que estavam dispostos a falar do seu cão. Medidas objetivas de impacto numa amostra aleatória talvez pudessem indicar outros resultados.

\section{CONCLUSÃO}

Em nosso estudo, concluímos que os proprietários de cães consideraram que seu sono não é prejudicado pelo cão, dormindo no mesmo leito ou no mesmo quarto, apesar de apresentarem mais despertares.

\section{REFERÊNCIAS}

1.Vilà C, Savolainen $\mathrm{P}$, Maldonado JE, Amorim IR, Rice JE, Honeycutt $\mathrm{RL}$, et al. Multiple and Ancient Origins of the Domestic Dog. Science 1997;276:1687-9. https://doi.org/10.1126/science.276.5319.1687

2.Fita DS, Costa-Neto EM. As interações entre os seres humanos e os animais: a contribuição da etnozoologia. Biotemas 2007;20:99-110. https://doi.org/10.5007/\%25x

3.Ball H. Bed-sharing and co-sleeping. Durban Res Online 2009;48:22-7.

4. McGarvey C, McDonnell M, Hamilton K, O'Regan M, Matthews T. An 8 year study of risk factors for SIDS: bed-sharing versus non-bedsharing. Arch Dis Child 2006;91:318-23. https://doi.org/10.1136/adc.2005.074674

5.Kushida CA, Chang A, Gadkary C, Guilleminault C, Carrillo O, Dement WC. Comparison of actigraphic, polysomnographic, and subjective assessment of sleep parameters in sleep-disordered patients. Sleep Med 2001;5:389-96. https://doi.org/10.1016/S13899457(00)00098-8

6.Dormir com cães na mesma cama (endereço de internet). Brasil: Comissão de Animais de Companhia (última atualização 04/2010; citado em 05/ 2013). Disponível em: www.comacvet.org.br/comac/Default.aspx 
7. Bertocco BP, Carvalho LBC, Prado AJF, Prado LBF, Prado GF. Bed sharing with dogs and the influence in owners' sleep. Sleep 2012;35(Abs suppl):A446.

8. Friedmann E, Thomas AS. Pet Ownership, Social Support, and Oneyear Survival After Acute Myocardial Infraction in the Cardiac Arrhythmia Suppression Trial (CAST). Am J Cadiol1995;76:1213-7. https://doi.org/10.1016/s0002-9149(99)80343-9

9. Blair PS. Putting co-sleeping into perspective. J Pediatric 2008;84:99-101. http://dx.doi.org/10.1590/S0021-

75572008000200001

10.Silva AS, Carvalho LBC, Silva L, Medeiros M, Natale VB, Carvalho JEC, et al. Sleep Habits and Starting Time to School in Brazilian Children. Arq Neuropsiquiatr 2005;63:402-6.

http://dx.doi.org/10.1590/S0004-282X2005000300007

11.Breugelmans JG, Ford DE, Smith PL, Punjab NM. Differences in Patient and Bed Partner-assessed. Quality of life in Sleep-disordered Breathing. Am J Respir Critical Care Med 2004;170:547-52. https://doi.org/10.1164/rccm.200310-14210C

12.Sobralske MC, Gruber ME. Risks and benefits of parent/child bed sharing. J Am Acad Nurse Pract 2009;21:474-9. http://dx.doi.org/10.1111/j.1745-7599.2009.00430.x

13.Dement WC. History of Sleep Physiology and Medicine. In: Kryger $\mathrm{MH}$, Roth T, Dement WC. Principles and Practice of Sleep Medicine. 5 ed. Philadelphia: Elsevier Sauders, 2005; 1-12.

14.Pankhurst FP, Horne JA. The influence of bed partners on movement during sleep. Sleep 1994;17:308-15. http://dx.doi.org/10.1093/sleep/17.4.308

15.Sobralske MC, Gruber ME. Risks and benefits of parent/child bed sharing. J Am Acad Nurse Pract 2009;21:474-9. http://dx.doi.org/10.1111/j.1745-7599.2009.00430.x

16.Ferreira SA, Sampaio IB. Human-Animal Relationship and Welfare of the domiciled dog. Arch Vet Sci 2010;15:22-35. http://dx.doi.org/10.4415/ANN 11_04_12

17.Vacari AMH, Almeida FA. The importance of pets' visit in recovery of hospitalized children. Einstein 2007;5:111-6.

18. Bayne K. Development of the human-research animal bond and its impact on animal well-being. ILAR 2002;43:4-9. https://doi.org/10.1093/ilar.43.1.4

19.Lima JVRBC. Os indivíduos e seus bichos na cidade de Brasília. Rev Soc Hum 2009;22:747-68.

20.Aiba N, Hotta K, Yokoyama M, Wang G, Kamiya K, et al. Usefulness of Pet Ownership as a Modulator of Cardiac Autonomic Imbalance in Patients With Diabetes Mellitus, Hypertension, and/or Hyperlipidemia. Am J Cardiol 2012;109:1164-70. https://doi.org/10.1016/j.amjcard.2011.11.055

21.Virues-Ortega J, Barriuso RP, Castellote JM, Pablacion A, Cuesta JP. Effect of Animal-assisted Therapy on the Psychological and functional status of elderly populations and patients with psychiatric disorders: a meta-analysis. Health Psychol Rev 2012;6:197-221. 
https://doi.org/10.1080/17437199.2010.534965

22.Braun C, Stangler T, Narveson J, Pettingell S. Animal-assisted therapy as a pain relief intervention for children. Complem Ther Clin Pract 2009;15:105-9. https://doi.org/10.1016/j.ctcp.2009.02.008 23. Chomel BB, Sun B. Zoonoses in the Bedroom. Emerging Infectious Diseases 2011;17:167-72. https://doi.org/10.3201/eid1702.101070 